\title{
Functional Regeneration of Chronically Injured Sensory Afferents into Adult Spinal Cord after Neurotrophin Gene Therapy
}

\author{
Mario I. Romero, ${ }^{2}$ Nagarathnamma Rangappa, ${ }^{1}$ Mary G. Garry, ${ }^{2}$ and George M. Smith ${ }^{1}$ \\ 1Department of Physiology, Spinal Cord and Brain Injury Research Center, University of Kentucky, Albert B. Chandler \\ Medical Center, Lexington, Kentucky 40536-0298, and 2Department of Anesthesiology and Pain Management, University \\ of Texas Southwestern Medical Center, Dallas, Texas 75390-9068
}

Lesioned axons within the dorsal roots fail to regenerate through the peripheral nerve transition zone and into the spinal cord. This regenerative failure leads to a persistent loss of sensory function. To induce axonal growth across this barrier, we used recombinant adenovirus to express fibroblast growth factor-2 (FGF2), nerve growth factor (NGF), L1 cell adhesion molecule (L1), or $\beta$-galactosidase (LacZ) within the endogenous glia of the dorsal spinal cord $16 \mathrm{~d}$ after injury. Expression of either FGF2 or NGF, but not L1 or LacZ, induced robust axonal regeneration into normal as well as ectopic locations within the dorsal spinal cord. This regeneration led to near-normal recovery of thermal sensory function. Functional recovery and the majority of regenerating axons within the dorsal horn disappeared with recutting of the sensory roots. Injections of adenovirus encoding NGF, but not FGF2, also resulted in extensive sprouting of noninjured sensory axons, which we previously demonstrated could cause hyperalgesia and chronic pain. Thus, neurotrophic factor gene therapy administered as late as $16 \mathrm{~d}$ after injury may serve as a useful treatment to elicit recovery after dorsal root avulsion; however, the choice of neurotrophin is important to induce selective regeneration of damaged axons.

Key words: gene therapy; regeneration; neurotrophins; functional recovery; spinal cord; adenovirus
Sensory modalities such as pain and proprioception are conveyed to the spinal cord by primary sensory neurons of the dorsal root ganglia (DRG), which send their axonal projections through the dorsal roots. After injury these axons regenerate within the peripheral nerve but stop abruptly at the CNS border, the dorsal root entry zone (DREZ). The inability of these axons to reinnervate the spinal cord results in permanent sensory loss and some motor control impairments (Reier et al., 1983; Carlstedt, 1985). The physiological mechanisms that prevent the growth of axons through the DREZ remain unidentified (Liuzzi and Lasek, 1987; Golding et al., 1999), although increases in chondroitin sulfate proteoglycans and reactive astrogliosis at the DREZ have been reported (Pindzola et al., 1993; Sims and Gilmore, 1994). Chondroitin sulfate proteoglycan production at the DREZ, however, is highly dependent on the severity of the injury and disruption of the blood-brain barrier (Finch and Silver, 1997). Within the adult spinal cord abortive regeneration may be caused by the presence of growth-inhibitory factors associated with myelin (Schwab and Bartholdi, 1996), reactive astrocytes (Davies et al., 1999), and a

Received Jan. 11, 2001; revised July 27, 2001; accepted July 30, 2001.

This study was supported by the J. F. Maddox Foundation, National Institute of Neurological Disorders and Stroke Grants NS33776 and NS38126 (to G.M.S.) and GM58057 (to M.G.G.), the Daniel Heumann Spinal Cord Foundation (M.I.R.), and the Sid W. Richardson Foundation. The expert technical assistance of Michael Davis, Jason Hale, and Martha Romero is greatly appreciated. We also appreciate a statistical examination of the data by Dr. J. F. Zolman.

Correspondence should be addressed to Dr. George M. Smith, Department of Physiology, MS 508, University of Kentucky, Albert B. Chandler Medical Center, Lexington, KY 40536-0298. E-mail: gmsmith@pop.uky.edu.

R. I. Romero's present address: Center for Developmental Biology, University of Texas Southwestern Medical Center, Dallas, TX 75390.

M. G. Garry's present address: Internal Medicine, University of Texas Southwestern Medical Center, Dallas, TX 75390.

Copyright (ㄷ) 2001 Society for Neuroscience $\quad 0270-6474 / 01 / 218408-09 \$ 15.00 / 0$ lack of growth-supporting molecules (Varon and Conner, 1994) However, the intrinsic growth state of injured neurons appears implicit for successful axonal regeneration (Chong et al., 1999; Neumann and Woolf, 1999).

Some of the strategies aimed at reversing the nonpermissive nature of the adult CNS attempt either to block inhibitory molecules (Schnell and Schwab, 1990) or to improve the regenerative conditions by using grafts of growth-supportive glia (Guest et al., 1997; Li et al., 1997) or genetically induced fibroblasts (Nakahara et al., 1996). In addition, glial expression of the cellular adhesion molecule L1 has been demonstrated to influence axonal regeneration in the optic nerve and spinal cord (Mohajeri et al., 1996). The direct administration of neurotrophic factors or acidic fibroblast growth factor also has been shown to increase axonal regeneration into the spinal cord after rhizotomy (Oudega and Hagg, 1996; Ramer et al., 2000) or after complete spinal cord transection by using peripheral nerve transplants (Cheng et al., 1996). Neurotrophins could act to increase the intrinsic neuronal growth state by downregulating the growth cone response to inhibitory signals (Cai et al., 1999) and increasing the degradation of extracellular inhibitory factors by the secretion of metalloproteinase (Zuo et al., 1998).

The use of in vivo gene therapy represents a feasible method to induce robust expression of growth-promoting molecules within an endogenous cell population in a spatially and temporally restricted manner (Smith and Romero, 1999). For this study we used recombinant adenoviruses to express conditionally nerve growth factor (NGF), fibroblast growth factor-2 (FGF2), and L1 within the dorsal spinal cord to analyze the regeneration of sensory axons after rhizotomies. Long-term expression of either NGF or FGF2 demonstrates extensive axonal regeneration and functional recovery of thermal nociception. 


\section{MATERIALS AND METHODS}

\section{Construction of adenoviral vectors}

Replication-defective recombinant adenoviruses were constructed as described previously (Romero and Smith, 1998; Romero et al., 2000). The functional characterization and spinal cord expression of these adenoviruses encoding LacZ, FGF2 (Dr. A. Baird, Selective Genetics, Inc., San Diego, CA), L1 (Dr. W. B. Stallcup, Burnham Institute, La Jolla, CA), and NGF (Regeneron Pharmaceuticals, Tarrytown, NY) have been reported previously (Romero et al., 2000). All plaque-purified adenoviruses were examined for replication-competent adenoviruses (RCA) via PCR. Then these viruses were amplified and purified by double cesium chloride gradient ultracentrifugation. The physical number of viral particles was determined by optical absorbency. The number of infectious particles was estimated by crystal violet staining via the agarose overlay method. The titers of these viruses ranged from 100 to 250 particle/ plaque-forming unit ( $\mathrm{pfu}$ ) ratios.

\section{Surgery and adenovirus administration}

In total, 43 adult (250-350 gm) female Sprague Dawley rats (Harlan Sprague Dawley, Indianapolis, IN), distributed among three independent experiments, were included in this study. Five experimental groups were tested: LacZ $(n=9), \operatorname{FGF}-2(n=13), \operatorname{NGF}(n=10), \operatorname{L1}(n=6)$, and FGF/11 $(n=5)$. Figure 1 illustrates both the injury and injection method. Briefly, deeply anesthetized animals [ketamine $(67 \mathrm{mg} / \mathrm{kg}$, i.p.)/ xylazine $(6.7 \mathrm{mg} / \mathrm{kg}$, i.p.)] underwent a hemilaminectomy at the L1-L2 vertebral segments to expose the lumbar dorsal roots. With the use of Drummond forceps, triple-crush lesions of $10 \mathrm{sec}$ each were inflicted at two sites separated by $3 \mathrm{~mm}$ along the L4-L5 afferents at $\sim 5-8 \mathrm{~mm}$ from the DREZ. Several randomly selected animals were examined $5 \mathrm{~d}$ after crushing to determine whether any axons survived the crush procedure. No axons were identified in the L4-L5 dorsal roots at the DREZ by either immunohistochemistry (see Fig. $1 B$ ) or transmission electron microscopy. The dorsal roots immediately rostral (L3) and caudal (L6) to the injured site were transected and ligated to prevent collateral sprouting. All lesions were performed unilaterally on the right side.

At 2 weeks after injury those animals with complete loss of sensory function of the affected hindlimb underwent a second hemilaminectomy at the T13-L1 vertebral segments to expose the lumbar spinal cord. All spinal cord microinjections were performed as described previously (Romero and Smith, 1998; Romero et al., 2000). Before the adenoviral administration the animals received $100 \mu \mathrm{g}$ intraperitoneally of a combined solution of rat CD-4 (W3/25) and CD-45 (MRC OX-22) antisera to suppress the immune system transiently. Each animal then received eight injections $(0.4 \mu \mathrm{l} ; 0.5 \mathrm{~mm}$ apart and $0.5 \mathrm{~mm}$ deep) of individual adenoviral vectors $\left(7.5 \times 10^{6} \mathrm{pfu} / \mu \mathrm{l}\right)$ along the L4-L5 DREZ. Dorsal musculature was sutured, and the skin incision was closed. Behavioral testing was performed every $8 \mathrm{~d}$ after the adenoviral injections.

For relesion experiments, dorsal roots L4 and L5 were reexposed. In these animals a small Silastic tube was placed around L4-L5 after the initial crushing for easy identification of these roots with reexposure. The tube was removed from L4 and L5, and the roots were cut and ligated to prevent any axonal regeneration. The wound was closed, and the rats were allowed to recover. All surgical procedures and animal maintenance comply with the regulations outlined by the Institutional Animal Care and Research Advisory Committee.

\section{Behavioral analyses}

Latency of paw withdrawal. The latency of paw withdrawal from a radiant heat source was used to measure the rats' response to noxious thermal stimuli, as previously described (Hargreaves et al., 1988). All animals were tested before adenoviral injections to establish baseline latencies, after which testing was performed every $8 \mathrm{~d}$. To describe the testing briefly, we placed the rats beneath an inverted clear plastic chamber on a glass floor. After a $5 \mathrm{~min}$ habituation period the plantar surface of the paw was exposed to a beam of radiant heat applied through the glass floor. Paw withdrawal latency (PWL) was detected automatically by a photocell and was taken as a behavioral index of the nociceptive threshold. Therefore, a score that was increased significantly over baseline represented analgesia (antinociception). A $20 \mathrm{sec}$ maximum was used to assure that no tissue damage occurred to the paw. Individuals conducting these experiments were always blinded as to the treatment. PWL readings were always taken in duplicate at $\sim 10 \mathrm{~min}$ intervals. Behavioral assessments of hyperalgesia and chronic pain were completed as described previously (Romero et al., 2000). Statistical analyses were per- formed with BMDP Statistical Software (Los Angeles, CA). Raw data (seconds \pm SEM) were analyzed by using multi-way ANOVA with repeated measures to determine the effects of treatment over time. Dunnett's post hoc analysis was used with an alpha of 5 .

Grid walking. Behavioral assessment for regeneration of proprioceptive axons was done by measuring the accuracy of paw placement on a grid runway as described by Kunkel-Bagden et al. (1993). Before lesioning all rats were trained to walk across a 6-foot grid runway, within which pegs were placed randomly $4-8 \mathrm{~cm}$ apart, for a food reward (peanut butter). Before and after lesioning each rat was videotaped making four complete crossings per trial. Foot placement was scored by analyzing the video in slow motion. Individuals conducting these experiments were always blinded as to the treatment. Accurate placement of the hindpaw on the pegs was scored as a positive $(+)$ and a misplacement (foot falls through the grid) was scored as an error $(-)$. The percentage of correct foot placements per trial was calculated and averaged for the four trials.

\section{Immunocytochemistry}

Tissue sections were incubated with polyclonal antiserum against rat calcitonin gene-related peptide (CGRP; 1:20,000; Sigma, St. Louis, MO). The CGRP antibody recognizes rat and human CGRP and rat and human $\beta$-CGRP. There is no cross-reactivity with substance $\mathrm{P}$, vasoactive intestinal peptide, calcitonin, somatostatin, or neuropeptide Y. Specific staining in rat tissue is inhibited by antiserum preincubation with 10 $\mu \mathrm{M}$ rat CGRP. Exclusion of any of the primary antisera from the staining protocol rendered no specific staining. Tissue sections of both control and experimental groups were developed simultaneously by using identical incubating solutions. Sections for each sample also were double labeled against anti-CGRP and goat anti-choline acetyltransferase (1: 2000; Chemicon, Temecula, CA). Visualization was achieved by tissue incubation in fluorescent or biotinylated secondary antibodies. Biotinlabeled tissue was processed further with the Vectastain Elite $A B C$ reagents (Vector Laboratories, Burlingame, CA) and developed by using two peroxidase substrate kits for brown and blue color (Vector SG). All images were captured with a PentaMax 16-bit CCD video camera connected to a Nikon E-800 epifluorescent microscope.

\section{Image analysis}

Axonal growth from digital images was quantified by MetaMorph Image Analysis software (Universal Imaging, West Chester, PA). Images from two randomly selected regions within each of the four lumbar segments (L3, L4, L5, and L6) per animal were quantified densitometrically. This was done by first applying a standardized optical density threshold to each image and then by measuring the area, in square micrometers, occupied by density profiles equal to or greater than the threshold. For each section the measurements were taken from the dorsal horn lateral to midline and above the plane of the spinal canal. With the use of this method it was possible to measure accurately the area occupied by CGRP-positive axons within the right dorsal quadrant of each spinal cord section. Raw data were analyzed by ANOVA with repeated measures. Significant interactions were evaluated, when appropriate, by a simple effects ANOVA, followed by a Tukey-Kramer post hoc test (Zolman, 1993).

\section{RESULTS}

\section{FGF2- and NGF-induced extensive axonal growth in the chronically denervated dorsal spinal cord}

For these experiments dorsal roots L4 and L5 were lesioned by triple crushes at two separate sites, severing all sensory axons within these roots (Fig. 1 $A, B$ ). To reduce sprouting from adjacent segments, we cut and tied the L3 and L6 roots to prevent axonal regeneration. This paradigm effectively denervated sensory input into the dorsal spinal cord from L3 to L6. During the first 2 weeks postlesion the animals were tested behaviorally to confirm the completeness of the L3-L6 rhizotomy. On day 16 all rats unilaterally received injections of adenovirus encoding $\mathrm{LacZ}$ (LacZ/ Adts), NGF (NGF/Adts), FGF2 (FGF2/Adts), or L1 (L1/Adts). Eight injections in total were made into the dorsal horn along the DREZ of L4 and L5 (Fig. 1 $A, C$ ). Histological examination of spinal cords 4 weeks after injection of LacZ/Adts (control) showed an almost complete loss of CGRP-containing fibers 

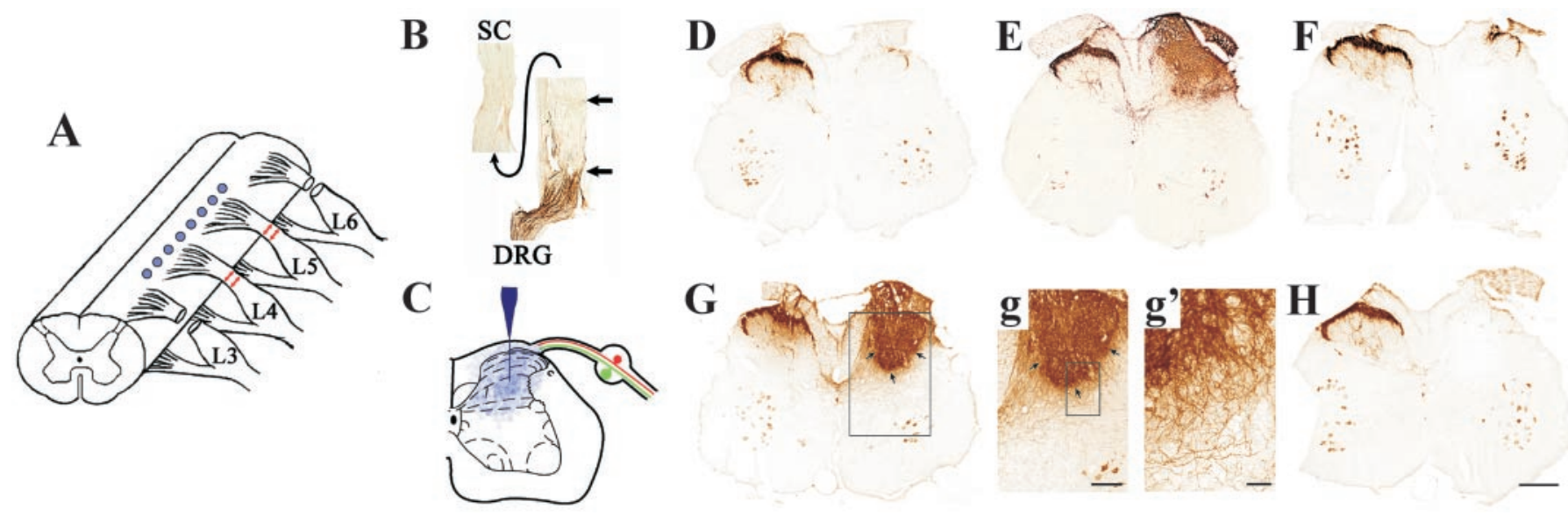

Figure 1. A, Illustration of the experimental paradigm showing the lesioned roots and adenoviral injection pattern. Dorsal roots L3 and L6 were cut and tied to prevent collateral sprouting, whereas dorsal roots L4 and L5 were lesioned by triple crushing at two separate sites (red lines). B, Crushing technique resulted in the degeneration of all neurofilament-positive axons distal (SC) to the crush sites (arrows). Then 2 weeks after the lesions the animals received eight microinjections along L4-L5 (circles in $A$ ). $C$, Illustration showing adenovirus injections into the dorsal horn at a depth of $0.5 \mathrm{~mm}$. $D-H$, Photomicrographs of the dorsal horn of the spinal cord showing the localization of immunoreactive CGRP primary afferents at L4-L5 in chronically injured rats treated with LacZ/Adts $(D), \mathrm{NGF} / \operatorname{Adts}(E), \mathrm{L} 1 /$ Adts $(F)$, FGF2/Adts $\left(G, g, g^{\prime}\right)$, or L1/FGF/Adts $(H)$ at $32 \mathrm{~d}$ after treatment. Both FGF2 and NGF induced extensive regeneration of CGRP-positive fibers throughout the injected dorsal horn. Axonal regeneration is very dense in the dorsal region near the area of transgene expression surrounded by a margin of reduced growth (arrows). $g$, $g^{\prime}$, High magnifications of the boxed inset in $G$ show the extent of axonal growth throughout the entire dorsal horn, with individual axons clearly visible in the area of reduced growth. Surprisingly, the combination of L1 and FGF2 $(H)$ resulted in a complete lack of growth of CGRP-positive fibers into the spinal cord. Scale bars: $D-H$, $0.5 \mathrm{~mm} ; g, 250 \mu \mathrm{m} ; g^{\prime}, 50 \mu \mathrm{m}$.

within the dorsal horn of L3-L6 (Figs. $1 D, 2 B, 4 A$ ) when compared with normal nonlesioned controls (Fig. $2 A$ ). In LacZ/Adtsinjected spinal cords numerous CGRP-positive axons were observed within the dorsal root terminating at the DREZ (Fig. $2 B$ ). In most cases a limited number of CGRP-positive fibers also were visualized within the dorsolateral tract of Lissauer (Fig. 2B). A few animals showed some fibers within the superficial layer of lamina I (Figs. 1D, 2B), representing collateral fibers from regions either below L6 or above L3; however, these axons failed to extend very far into the dorsal horn. Similarly, none of the spinal cords injected with L1/Adts showed an increase in the number of CGRP-positive fibers (Fig. $1 F$ ).

The vast majority of chronically denervated animals that received either FGF2/Adts (Figs. $1 G, 2 C$ ) or NGF/Adts (Figs. $1 E$, $2 D)$ showed a dramatic increase in the detectable numbers of CGRP-positive fibers regenerating into the dorsal horn. In both FGF2- and NGF-treated groups sensory afferents were observed in normal (laminas II and IV) as well as abnormal (laminas III, VI, and VII-X in the ventral horn) target areas (Fig. 1E,G). In these animals sensory fibers were found throughout the entire dorsal horn, growing within both gray and white matter. The overexpression of either FGF2 or NGF within the dorsal horn supported robust growth of CGRP-positive axons across the DREZ, through the cuneate fasciculus, and into the dorsal horn (Fig. $2 C, D$ ). Axonal growth was markedly dense within the region surrounding the injection sites, which correlates with the localization of transgene expression observed in our earlier studies (Romero and Smith, 1998; Romero et al., 2000). The area of dense axonal growth appeared circumscribed, with a sharp decline in the number of CGRP-positive fibers (Figs. 1, 3). Many of the fibers outside this region grew toward the central canal or into laminas VII and IX in the ventral spinal cord.

Previous studies have demonstrated that L1 is a potent promoter of neurite growth, which mediates growth via the FGF receptor as well as other pathways. Because L1 alone did not induce regeneration of CGRP axons through the DREZ, we hypothesized that the coexpression of this cellular adhesion molecule with a neurotrophic factor, particularly FGF2, would augment neurite growth. Contrary to this hypothesis, the coadministration of L1/Adts and FGF2/Adts (see Fig. $1 H$ ) in five of five rats showed no axonal regeneration when compared with LacZ/ Adts controls (compare Fig. $1 D, G$ with $H$ ), indicating that L1 negatively regulates FGF2-induced axonal growth. Similar results also have been observed with the coexpression of NGF (our unpublished observations).

\section{Expression of CGRP within spinal cord neurons}

Does the overexpression of NGF dramatically modulate neuropeptide levels in trkA-positive neurons within the spinal cord, causing increased endogenous CGRP expression? In addition, FGF2 may elicit this effect also, because overexpression of FGF2 upregulates the release of NGF from astrocytes (Yoshida and Gauge, 1992; Romero et al., 2000). To examine this possibility further, we double-labeled spinal cord sections for CGRP and choline acetyltransferase (ChAT). The majority of trkA-positive neurons within the adult spinal cord coexpresses ChAT (Michael et al., 1997), and the majority of ChAT-expressing motor neurons also expresses CGRP (New and Mudge, 1986). Increases in CGRP expression within the dendrite of these neurons could contribute to the overall increase in CGRP-positive fibers observed within FGF2/Adts or NGF/Adts-treated animals. Evaluation of sections double-labeled for CGRP and ChAT demonstrated no increase in CGRP within the dendrites or somas of ChAT-positive neurons (Fig. $2 E-H$ ). In spinal cords injected with LacZ/Adts, ChAT-positive neurons were observed within laminas VII and IX (Fig. 2E). Although none of the neurons within lamina VII expressed CGRP, the majority of the motor neurons within lamina IX did. CGRP staining, however, was not observed within the dendrites of these neurons. Spinal cords injected with NGF/Adts showed an identical ChAT staining pattern, with no 


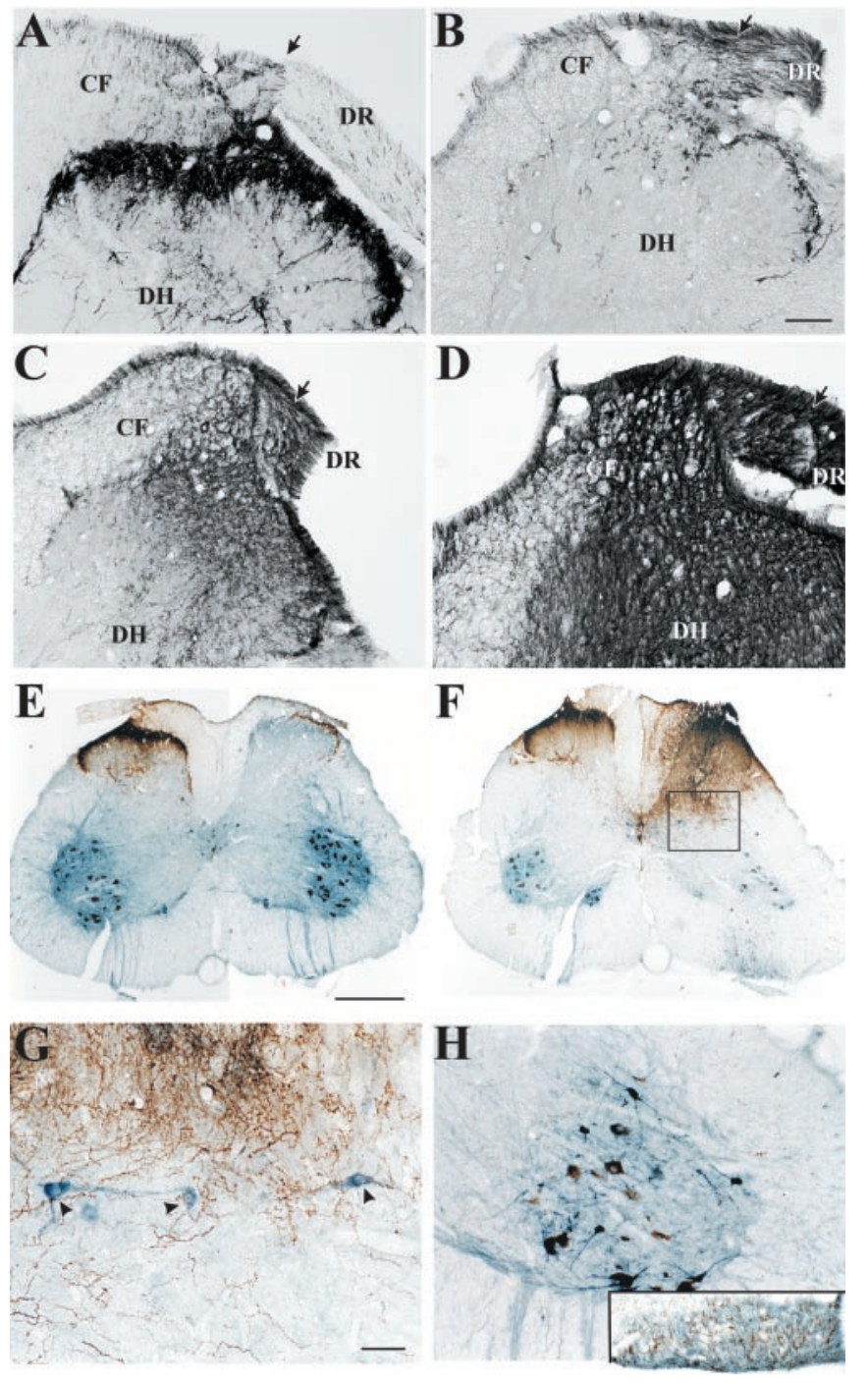

Figure 2. CGRP expression within the dorsal horn after FGF2/Adts or $\mathrm{NGF} /$ Adts treatment is attributable to the regeneration of sensory axons through the dorsal root entry zone (DREZ) and is not attributable to the upregulation of CGRP in dendrites or soma of neurons within the spinal cord. $A$, In the normal spinal cord CGRP-positive axons within the dorsal root $(D R)$ enter the cord through the DREZ (arrow). These fibers then traverse the cuneate funiculus $(C F)$ and terminate in laminas I and II of the dorsal horn $(D H)$. B, At $48 \mathrm{~d}$ after rhizotomy, followed by injections of LacZ/Adts, CGRP-positive axons failed to grow through the DREZ and into the spinal cord. A small contingent of fibers remains in Lissauer's tract (asterisks). Robust regeneration of CGRP-positive axons was observed extending through the DREZ and into the dorsal spinal cord after treatment of rhizotomized rats with either FGF2/Adts $(C)$ or NGF/Adts $(D)$. Double-labeling experiments for CGRP (brown) and choline acetyltransferase (ChAT; blue) further demonstrate that the majority of trkAexpressing neurons and dendrites within the dorsal horn does not express CGRP $(E-H)$. Low magnification of LacZ/Adts-injected $(E)$ and NGF/ Adts-injected $(F)$ spinal cords shows no increase in the overall number of ChAT-positive neurons but a major increase in CGRP staining. $G$, High magnification of boxed inset in $F$ shows three ChAT-positive neurons (arrowheads) that do not express CGRP in either their somas or dendrites; however, many CGRP-positive axons can be observed growing around them. CGRP-positive staining can be observed in the majority of motor neurons from normal, LacZ/Adts-treated $(E)$, or NGF/Adtstreated $(F, H)$ animals. Although the cell bodies of motor neurons express CGRP, no CGRP is apparent in motor neuron dendrites $(H)$. CGRPpositive axons can be observed within the ventral nerve of spinal cord that has been injected with NGF/Adts (inset in $H$ ). Scale bars: $A-D, 100 \mu \mathrm{m}$; $E-F, 0.5 \mathrm{~mm} ; G-H, 50 \mu \mathrm{m}$.
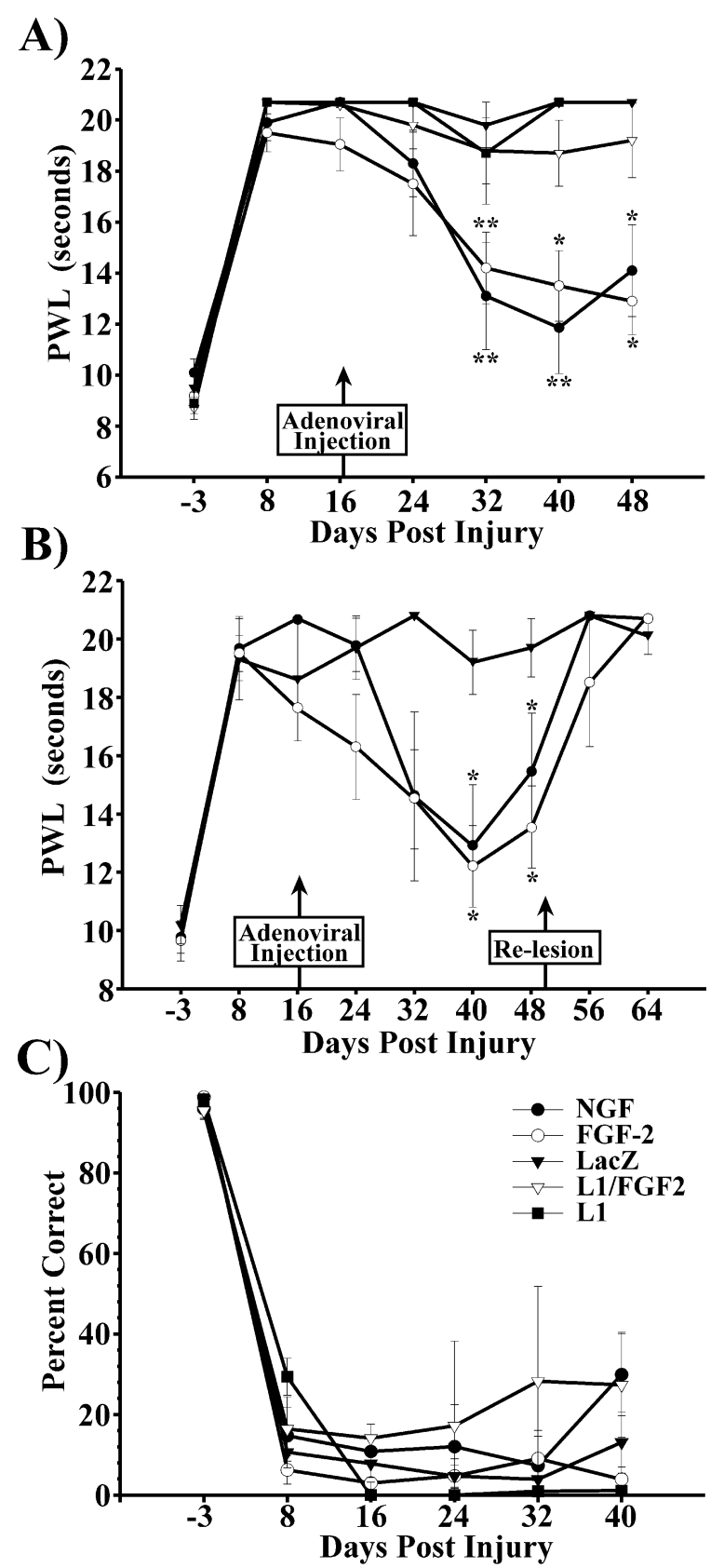

Figure 3. Regeneration of CGRP-positive axons induces functional recovery of thermal nociception. To verify successful lesioning of dorsal roots L3 through L6, we evaluated rats $3 \mathrm{~d}$ before $(-3)$ and 8 and $16 \mathrm{~d}$ after lesioning. $A$, At $16 \mathrm{~d}$ after lesioning and before adenoviral injections, all rats demonstrated a complete loss of thermal nociception ipsilateral to injury. Within 2 weeks after FGF2/Adts and NGF/Adts injections, functional recovery was apparent, and significant reductions in paw withdrawal latencies $(P W L)$ were observed. No significant change in PWL was observed with L1/Adts or L1/FGF2/Adts when compared with LacZ/ Adts-treated animals. $B$, In a separate group of animals, relesioning dorsal roots L4 and L5 resulted in the loss of functional recovery and a return to the PWL times similar to those of LacZ/Adts controls. Arrows indicate the time of Ad injections and relesioning. $C$, To examine functional recovery of sensory axons involved in coordinating leg positioning (proprioception), we also tested rats for errors in paw placement on a grid runway. No significant functional restoration in proprioception was observed in any of the tested groups. Data are presented as the mean \pm $\mathrm{SEM} ; n=4-6$ rats/group. ${ }^{*} p<0.05 ;{ }^{* *} p<0.01$. 


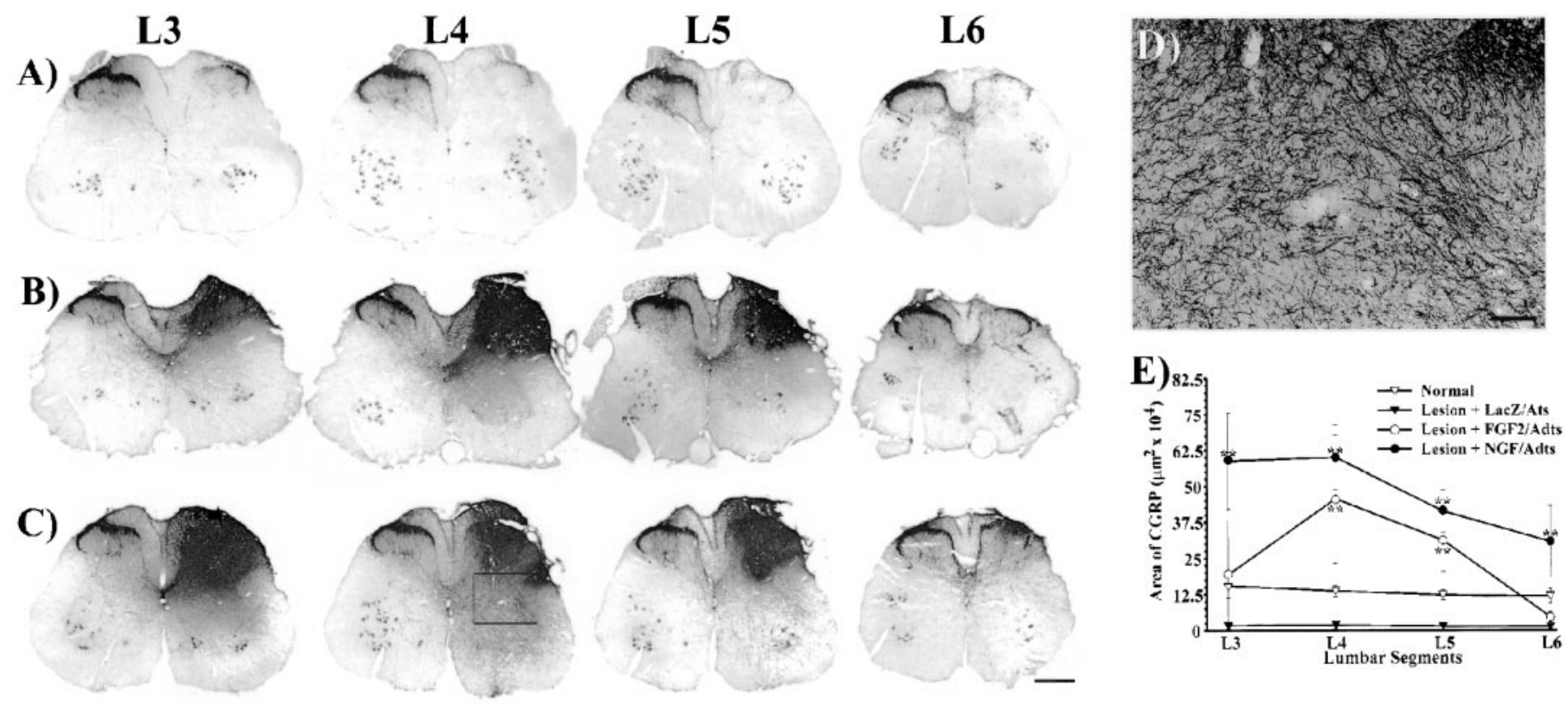

Figure 4. Photomicrographs of spinal cord segments L3, L4, L5, and L6 from animals treated with LacZ/Adts $(A)$, FGF-2/Adts $(B)$, or NGF/Adts $(C)$ at $48 \mathrm{~d}$ after rhizotomy. Compared with animals treated with LacZ/Adts, rats treated with either FGF-2/Adts or NGF/Adts showed a marked increase in immunoreactive CGRP-containing afferents, particularly at the injection site (L4-L5). Dense CGRP-positive fiber growth also was identified in L3 and L6 segments in spinal cords injected with NGF/Adts. D, High magnification of the boxed inset from L4 spinal segment in $C$. E, Quantitative analyses of treated spinal cords demonstrate a 10-fold loss in the area occupied by CGRP-positive axons after rhizotomy and injection of LacZ/Adts when compared with normal CGRP expression. Rhizotomy and injection of either FGF2/Adts or NGF/Adts resulted in a $>25$-fold increase (when compared with lesion + LacZ/Adts) in the area of the dorsal spinal cord occupied by CGRP-positive axons. Data are presented as the mean \pm STD; ** $p>0.001$. Scale bars: $A-C, 0.5 \mathrm{~mm} ; D, 100 \mu \mathrm{m}$.

apparent increase in ChAT cell number or colocalization with CGRP (Fig. $2 F-H$ ). Within lamina VII of these animals ChATpositive neurons and dendrites did not colabel with CGRP, but numerous CGRP-positive axons could be observed extending around ChAT-positive neurons (Fig. 2G). Furthermore, in all sections stained with CGRP we never observed a staining pattern reminiscent of neuronal cell bodies or dendrites within the dorsal horn, but we consistently observed axon-like structures (Figs. $\left.1 g, g^{\prime}, 2 C, D, G\right)$. Similar to LacZ/Adts-treated animals, NGF/Adts showed CGRP-positive staining of motor neurons, but not their respective dendrites (Fig. $2 H$ ). In many NGF/Adts-treated animals CGRP-positive fibers also were observed within the ventral funiculus extending into the ventral root, most likely consisting of axons from CGRP-positive motor neurons (Fig. 2).

\section{FGF2- and NGF-induced recovery of nociceptive function}

The functional significance of the anatomical findings reported above was evaluated in a double-blinded design, using the plantar heat test and a grid runway to test nociception and proprioception, respectively. Hindlimb nociception was evaluated by measuring PWL to a noxious thermal stimulus. Normal animals remove their paw from this stimulus within $8-10$ sec. At 1 and 2 weeks after the L3-L6 dorsal rhizotomy the animals showed a complete loss of thermal sensation ipsilateral, but not contralateral, to the lesions. At $16 \mathrm{~d}$ after rhizotomy the spinal cords were injected with LacZ/Adts, FGF2/Adts, L1/Adts, NGF/Adts, or FGF2-L1/Adts into L4 and L5 segments. Rats treated with LacZ/ Adts, L1/Adts, or a combination of L1/Adts-FGF2/Adts demonstrated no functional recovery of thermal nociceptive or proprioceptive responses (Fig. 3). Conversely, both FGF2/Adts- and NGF/Adts-treated animals showed gradual recovery of nocicep- tive function. This effect was observed first as early as 1 week after injection, becoming statistically significant and reaching near-normal values 2-3 weeks after administration (Fig. 3A). Anatomical examination of the individual animals showed a distinct correlation between functional recovery and the regeneration of CGRP-positive axons, as demonstrated in Figure 4.

To verify that this functional recovery of thermal nociception was mediated by axons that had regenerated through the lesion, we relesioned dorsal roots L4 and L5 4 weeks after the adenoviral injections in a second series of experiments. Relesioning these dorsal roots completely eliminated thermal nociceptive responses in FGF2/Adts- and NGF/Adts-injected animals (Fig. 3B). These results strongly suggest that the functional recovery observed in animals that overexpressed FGF2 or NGF is mediated primarily by true regeneration of L4-L5 dorsal primary afferents.

To determine whether regeneration and functional recovery were specific to nociceptive axons, we examined the animals by using a grid runway to examine correct paw placement that is indicative of normal proprioceptive functioning (Fig. 3C). Nonlesioned animals exhibit correct hindpaw placement on individual rungs with each step. After injury, however, performance decreases to $<30 \%$ accuracy in foot placement. The overexpression of FGF2, L1, NGF, or FGF2/11 failed to demonstrate statistically significant increases in foot placement, indicating that the functional recovery observed after injecting FGF2/Adts and NGF/ Adts was specific for nociceptive axons.

\section{Axonal regeneration and collateral sprouting are induced by NGF, whereas FGF2 primarily stimulates axonal regeneration}

After functional recovery was observed, as shown in Figure $3 A$, the animals were examined histologically for axonal regeneration. 



Figure 5. Relesioning dorsal roots L4 and L5 resulted in an almost complete loss of CGRP-containing fibers within the L4 and L5 regions of the spinal cord. To verify that axons regenerated through dorsal roots L4 and L5, we examined the spinal cords of rats injected with either FGF2/Adts ( $A$ ) or NGF/Adts $(B)$ in Figure $3 B$ at $14 \mathrm{~d}$ after relesioning. Representative sections of spinal segments L3-L6 showed a dramatic reduction in the overall CGRP fiber pattern (compare with representative treatment in Fig. 4). Quantitative analyses show an $\sim 88$ and $57 \%$ reduction in area occupied by CGRP-positive axons in relesioned FGF2/Adts-treated $(C)$ and NGF/Adts-treated $(D)$ animals, respectively. Some CGRP-positive axons were identifiable in the L3 region of FGF2/Adts-injected rats, whereas extensive growth (approximately ninefold greater than FGF2/Adts + relesion) of these fibers was apparent in NGF/Adts-injected rats. Data are presented as the mean \pm STD; ${ }^{*} p>0.001$. Scale bar, $0.5 \mathrm{~mm}$.

Evaluation of L3-L6 segments in LacZ/Adts-treated animals concluded that there was no spontaneous regeneration or sprouting of CGRP-positive axons within the L4 and L5 spinal cord segments (Fig. 4A). Some collateral sprouting of noninjured fibers into lamina III was evident only at the border of L3 and L6 levels, although fibers were still present in Lissauer's tract at L4 and L5. In nonlesioned animals CGRP-positive fibers at the L4 and L5 spinal segments occupy $\sim 136,613 \pm 18,900$ and 122,075 \pm $17,307 \mu \mathrm{m}^{2}$ of the dorsal horn, respectively (Fig. $4 E$ ). Rhizotomy reduces CGRP fiber density by $\sim 10$-fold at L4 (16,853 $\pm 14,068$ $\left.\mu \mathrm{m}^{2}\right)$ and L5 $\left(13,053 \pm 11,218 \mu \mathrm{m}^{2}\right)$ (Fig. 4D). Expression of FGF2 (Fig. $4 B$ ) induced substantial growth of CGRP-positive axons, which were concentrated around the injection areas (L4L5). At these segments CGRP fiber density increased 27-fold $\left(458,351 \pm 223,836 \mu \mathrm{m}^{2}\right)$ and 24-fold $\left(312,900 \pm 107,853 \mu \mathrm{m}^{2}\right)$ when compared with LacZ/Adts-injected spinal cords. Fiber density at the L3 and L6 segments showed no statistically significant change from LacZ/Adts-treated rats (Fig. 4E). These data are reflective of axonal regeneration through the DREZ of L4 and L5 rather than sprouting from border segments. Animals injected with NGF/Adts showed comparable fiber growth at the L4 and L5 segments (Fig. 4C); however, numerous CGRP-positive fibers also were visualized at the L3 and L6 segments (compare Fig. $4 B, C$ at the L3 level). Within the spinal segments L 4 and L5 a relatively high density of CGRP-positive axons was observed extending through laminas VI and VII (Fig. 4D). Quantitative analysis of fiber density showed a $>25$-fold increase at all four segments when compared with LacZ/Adts-treated animals (Fig. 4E). At L4 and L5 CGRP-positive fibers occupied 603,699 \pm
113,332 and $415,197 \pm 75,249 \mu \mathrm{m}^{2}$. In addition to axonal regeneration through L4 and L5 DREZs, the distinct pattern of fiber growth in the NGF/Adts-treated animals suggests a concomitant increase in collateral sprouting at the L3-L6 segments.

The pattern of FGF2- or NGF-induced axonal growth observed along the L3-L6 lumbar cord suggests that they have differential effects in chronically denervated spinal cords. To discriminate further the axonal regeneration from collateral sprouting, we examined the relesioned animals described in Figure $3 B$ histologically. These animals were evaluated 2 weeks after dorsal roots L4 and L5 were recut. Primary afferents that originally had regenerated through the DREZs of L4 and L5 degenerated after relesioning, thereby causing the disappearance of CGRP-positive fibers attributed to regeneration through these roots. Relesioning of animals treated with FGF2/Adts (Fig. $5 A$ ) reduced the area of CGRP innervation by $88 \%$ at L4 $(54,265 \pm$ $\left.27,581 \mu \mathrm{m}^{2}\right)$ and $89 \%$ at L5 $\left(35,766 \pm 13,483 \mu \mathrm{m}^{2}\right)$. The relatively low density of CGRP fibers at L3 and L6 demonstrated no statistically significant reduction between regenerated and relesioned groups, suggesting little axonal growth into those segments (Fig. $5 C$ ). Similar experiments in animals treated with NGF/Adts showed 57 and $72 \%$ reductions in CGRP-positive fibers at the L4 $\left(259,701 \pm 130,036 \mu \mathrm{m}^{2}\right)$ and L5 $\left(115,865 \pm 48,233 \mu \mathrm{m}^{2}\right) \mathrm{seg}-$ ments, respectively (Fig. 5B,D). Within spinal segment L3 and L4 the CGRP-positive fibers were observed to a greater extent in NGF/Adts-treated than in FGF2/Adts-treated animals (compare relesioned groups in Fig. 5C,D). These data demonstrate that the injection of either FGF2/Adts or NGF/Adts induced robust regeneration of CGRP-positive fibers through the L4 and L5 

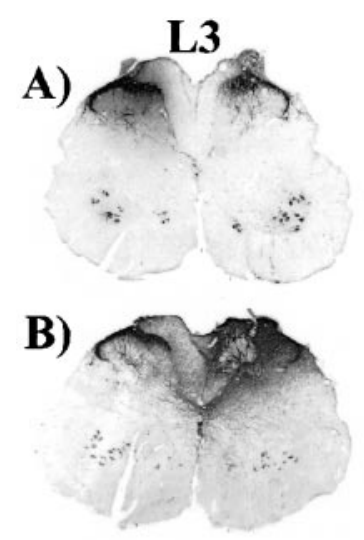
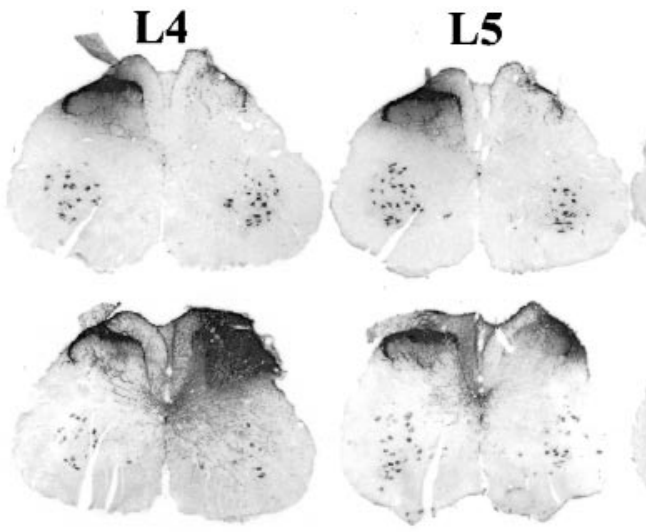
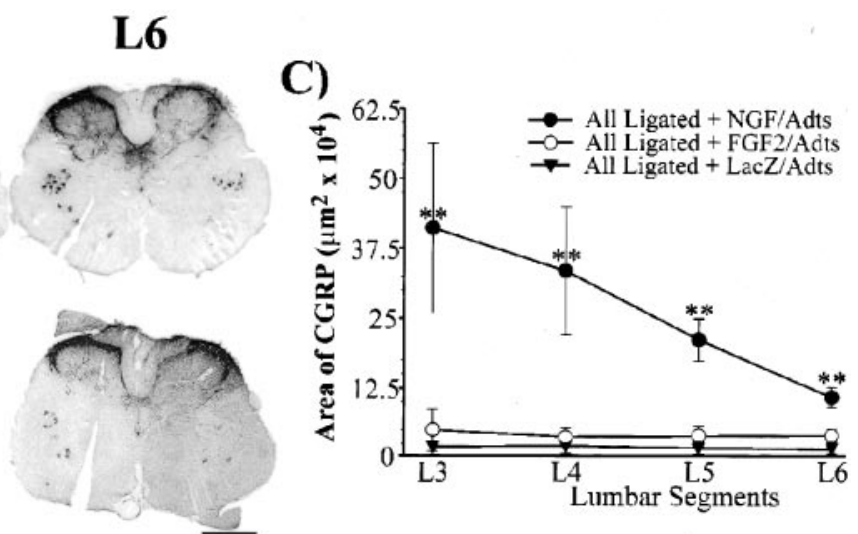

Figure 6. Injections of NGF/Adts, but not FGF2/Adts, resulted in robust collateral sprouting of CGRP-positive axons into denervated regions of the spinal cord. To examine the differences in the ability of FGF2/Adts $(A)$ and NGF/Adts $(B)$ to induce collateral sprouting, we cut and ligated dorsal roots L3-L6 to prevent axonal regeneration. $C$, Quantitative analyses of spinal cord sections L3, L4, L5, and L6 show little collateral sprouting 28 d after FGF2/Adts has been injected, when compared with LacZ/Adts. In contrast, extensive collateral sprouting is observed throughout all four spinal segments $28 \mathrm{~d}$ after injections of NGF/Adts. Data are presented as the mean \pm STD; ** $p>0.001$. Scale bar, $0.5 \mathrm{~mm}$.

DREZ. Additionally, NGF/Adts, but not FGF2/Adts, induced extensive collateral sprouting from noninjured dorsal afferents into the marginal denervated regions within the dorsal horn. These data also indicate that collateral sprouting induced by NGF did not contribute to the functional recovery, because all nociceptive responses were lost after dorsal roots L4 and L5 were relesioned in these animals.

To examine further the axonal spouting into denervated segments of the spinal cord after overexpressing either FGF2 or NGF, we severed and tied all four dorsal roots (L3-L6) to prevent axonal regeneration through those roots. As in the other experiments LacZ/Adts, FGF2/Adts, or NGF/Adts was injected at the DREZ of L4 and L5 2 weeks postlesioning. In all of the animals that were examined, treatment with FGF2/Adts (Fig. 6A) resulted in few CGRP-positive fibers within segments L3 through L6 and showed no statistically significant difference when compared with the LacZ/Adts-treated group (Fig. 6C). In contrast, animals treated with NGF/Adts showed substantial fiber growth throughout spinal segments L3-L6, strongly indicating that NGF, but not FGF2, acts as a potent inducer of axonal sprouting (Fig. 6B,C). Behavioral analyses showed that preventing axonal regeneration through dorsal roots L3-L6 blocked recovery of thermal nociceptive responses up to 4 weeks after injection, even in animals injected with either NGF/Adts or FGF/Adts.

\section{DISCUSSION}

These results demonstrate the effectiveness of neurotrophin gene therapy for inducing axonal regeneration within the spinal cord. The direct transfer and expression of neurotrophic genes exhibit multiple benefits over transplanting normal or genetically engineered cells by locally reducing the inhibitory nature of the adult CNS milieu. In addition, the extent of axonal regeneration observed was dramatically greater than that observed after the application of purified neurotrophins with the use of osmotic minipumps (Oudega and Hagg, 1996; Ramer et al., 2000). The density of regenerating axons appeared highest in regions associated with injections that correlated to the areas of transgene expression (Romero et al., 2000). This indicates that a more discrete pattern of growth might be established by carefully controlling the injection and expression of growth-promoting molecules within distinct locations, such as laminas I and II, instead of the entire dorsal horn. Experiments in which dorsal roots L4 and L5 were relesioned or ligated to prevent axonal regeneration indicated that damaged axons grew better than intact axons in the presence of FGF2, whereas in the presence of NGF both regeneration of damaged axons and sprouting of intact axons were observed. These results correlate with our earlier observation that in the noninjured spinal cord NGF, but not FGF2, induced robust sprouting of CGRP- and substance P-positive fibers, resulting in hindpaw-guarding behavior indicative of chronic pain syndromes (Romero et al., 2000). In the present study we observed the return of normal, protective pain, and none of the rats treated with either FGF2/Adts or NGF/Adts showed signs of hyperalgesia or chronic pain.

A recent study by Ramer et al. (2001) indicated that axons fail to regenerate through the dorsal white matter and into the dorsal horn with delayed intrathecal application of NT-3. The failure of axons to regenerate through this terrain was attributed to the presence of ED-1-expressing macrophages and the establishment of myelin debris. In our experiments the adenovirus injections were delayed $16 \mathrm{~d}$ after rhizotomy and resulted in robust regeneration of sensory afferents throughout the dorsal spinal cord, even into the degenerating white matter of the dorsal columns. The differences between the two studies, however, are numerous. Although Ramer et al. (2001) used osmotic minipumps to supply NT-3 to the intrathecal space surrounding the spinal cord, NT-3 penetration and concentration within the spinal cord were never determined. In addition, activated microglia/brain macrophages are known to secrete numerous proteases (Rosenberg et al., 2001) that could accelerate the degradation of NT-3 diffusing into the spinal cord. Injections of NGF/Adts result in expression levels 10 -fold greater ( $\sim 5 \mathrm{ng} \mathrm{NGF/mg} \mathrm{tissue)} \mathrm{than} \mathrm{those} \mathrm{measured} \mathrm{in}$ control spinal cords (Romero et al., 2000). Immunohistology further demonstrated that transgene expression was concentrated to the dorsal spinal cord extending up to the DREZ (Romero et al., 2000). Expression of these relatively high neurotrophin levels may elicit a stronger influence on growth cone migration through the nonpermissive CNS environment. Another difference between the two studies is that trkA is expressed at much higher levels than trkC within the normal adult DRG and after nerve injury (Bergman et al., 1999). This observation would suggest that, in the adult, trkA-positive neurons would regenerate better 
in the presence of NGF than trkC-positive neurons would in the presence of NT-3. Postlesion reductions in trkC expression might impair NT-3 mediated axonal regeneration.

The mechanisms by which NGF or FGF2 might induce regeneration are still unclear; however, recent studies demonstrate that the molecular constituents of the growth cone profoundly influence axonal growth and guidance (Ming et al., 1999). The growth cone turning response to a chemoattractive or repulsive factor is influenced directly by the activity of protein kinase A (PKA), the levels of cyclic nucleotide, or $\mathrm{Ca}^{2+}$ (Song et al., 1997, 1998). Increased levels or activation of these factors caused growth cones to grow toward a chemorepulsive factor, the exact opposite of its normal behavior. Priming of cerebellar or DRG neurons with specific neurotrophins also has a similar effect, allowing neurons to overcome the inhibitory influence of myelin or myelinassociated glycoprotein (Cai et al., 1999). Preexposure to neurotrophins caused an increase in the levels of cAMP and the activation of PKA. This effect appears highly dependent on the presentation of neurotrophin to growth cones, in which cytosolic signals responsible for altering growth cone behavior are desensitized by uniform exposure, but not by a gradient of neurotrophin (Ming et al., 1999). Gradients most likely are induced in the spinal cord defusing away from the adenovirally transduced cells expressing the neurotrophins. These data may provide a mechanism to explain why axonal density was the greatest near the injection sites.

Although both NGF and FGF2 induced axonal regeneration, their mechanisms of action may be very different. Initially, an NGF gradient was thought to act as a chemoattracting factor to guide growing axons to their targets (Gundersen and Barrett, 1980). Recent data, however, demonstrate that neurotrophins function as targeting factors in which they support invasion, elaboration, and axonal branching into target fields (Hoyle et al., 1993; ElShamy et al., 1996). Under these conditions NGF expression in the sensory terminal field of the spinal cord might induce sprouting from noninjured axons as well as regeneration from injured primary afferents. The competition between sprouting and regenerating axons may explain why fewer axons sprout into L4 and L5 regions in relesioned animals when compared with animals in which all four roots were cut and ligated.

\section{Regeneration, but not sprouting, is induced by FGF2}

Exogenously applied FGF2 is known to stimulate the survival and transmitter metabolism of CNS neurons both in vivo and in vitro (Anderson et al., 1988; Grothe et al., 1991). The activity of FGF2 may be mediated directly by FGF receptors (FGFR) that are found in ventral motor neurons and expressed in the intact DRG (Meisinger and Grothe, 1997). A possible role of FGF2 in the regeneration of DRG neurons has been suggested by the finding that injury to the sciatic nerve induced a rapid upregulation of FGF2 mRNA in injured neurons, from the basal level of $5 \%$ up to a level of $80 \% 3 \mathrm{~d}$ after injury (Grothe et al., 1997). Within the developing visual system FGF2 expression is confined to the optic tract, and perturbation experiments indicate its role in mediating axonal elongation (McFarlane et al., 1996). Furthermore, activation of the FGFR by the cell adhesion molecules NCAM, L1, or N-cadherin as well as FGF2, constitutes an important pathway for neurite growth (Williams et al., 1994). Axonal growth mediated by these adhesion molecules is abolished in transgenic mice expressing a dominant-negative form of the FGF receptor (Saffell et al., 1997). Our results show that the growth of CGRP-positive axons within the chronically denervated spinal cord in response to FGF2 is similar, but not identical, to that elicited by NGF. Although both NGF and FGF2 were able to induce robust growth of nociceptive fibers, only FGF2-treated animals showed almost a complete loss of innervation in the L3 and L6 border segments in all experimental groups. These results indicate that FGF2 does not elicit a strong sprouting influence on nondamaged CGRP-positive fibers but does enhance greatly the regeneration of these axons after injury. The ability of NGF to invoke aberrant sprouting can result in functional abnormalities such as chronic pain (Christensen and Hulsebosch, 1997) or autonomic dysreflexia (Krenz et al., 1999). Therefore, FGF2 treatment may be a better alternative to elicit more specific regeneration of severed axons after dorsal root avulsion.

\section{L1 blocks FGF2-induced axonal regeneration}

The expression of L1 in the dorsal horn elicited only modest growth of sensory fibers into the spinal cord. This result contrasts with the known capabilities of L1 to promote neurite growth in vitro (Mohajeri et al., 1996). Even more unexpected was the observation that coadministration of the L1 and FGF2 adenoviruses completely blocked the FGF2-induced regeneration, because it is known that the homophilic L1 interaction in neurons triggers the activation of neuronal FGF receptors (Williams et al., 1994). More recent studies have shown that L1 also inhibits NGF-induced regeneration and sprouting as well (our unpublished observations). The specific mechanism of this blockade is unknown; however, it is possible that the adherence of growing axons to the L1-expressing cells was stronger than the FGF2 signal to induce growth. Currently, we are investigating whether the L1 inhibition of FGF2-induced fiber growth can be mimicked by other cell adhesion molecules.

In summary, we demonstrated that in vivo gene transfer of FGF2 and NGF, but not L1, can induce axonal regeneration and functional recovery of chronically injured primary nociceptive fibers. The extensive axonal growth induced by this method supports the use of in vivo gene therapy as a means to deliver molecules aimed at transforming the adult CNS parenchyma from inhibitory to growth-promoting. In addition, the observation that NGF, but not FGF2, induced sprouting of noninjured fibers, suggests for the first time the possibility of using growth factors to induce the regeneration of injured axons without altering the current innervating pattern of noninjured ones.

\section{REFERENCES}

Anderson KJ, Dam D, Lee S, Cotman CW (1988) Basic fibroblast growth factor prevents death of lesioned cholinergic neurons in vivo. Nature 332:360-361.

Bergman E, Fundin BT, Ulfhake B (1999) Effects of aging and axotomy on the expression of neurotrophin receptors in primary sensory neurons. J Comp Neurol 410:368-386.

Cai D, Shen Y, De Bellard M, Tang S, Filbin MT (1999) Prior exposure to neurotrophins blocks inhibition of axonal regeneration by MAG and myelin via a cAMP-dependent mechanism. Neuron 22:89-101.

Carlstedt T (1985) Regenerating axons from nerve terminals at astrocytes. Brain Res 347:188-191.

Cheng H, Cao Y, Olson L (1996) Spinal cord repair in adult paraplegic rats: partial restoration of hind limb function. Science 273:510-513.

Chong MS, Woolf CJ, Haque NS, Anderson PN (1999) Axonal regeneration from injured dorsal roots into the spinal cord of adult rats. J Comp Neurol 410:42-54.

Christensen MD, Hulsebosch CE (1997) Spinal cord injury and antiNGF treatment results in changes in CGRP density and distribution in the dorsal horn in the rat. Exp Neurol 147:463-475.

Davies SJ, Goucher DR, Doller C, Silver J (1999) Robust regeneration of adult sensory axons in degenerating white matter of the adult rat spinal cord. J Neurosci 19:5810-5822.

ElShamy WM, Linnarsson S, Lee KF, Jaenisch R, Ernfors P (1996) Prenatal and postnatal requirements of NT-3 for sympathetic neuro- 
blast survival and innervation of specific targets. Development 122:491-500.

Finch MT, Silver J (1997) Activated macrophages and the blood-brain barrier: inflammation after CNS injury leads to increases in putative inhibitory molecules. Exp Neurol 148:587-603.

Golding JP, Bird C, McMahon S, Cohen J (1999) Behaviour of DRG sensory neurites at the intact and injured adult rat dorsal root entry zone: postnatal neurites become paralyzed, whilst injury improves the growth of embryonic neurites. Glia 26:309-323.

Grothe C, Wewetzer K, Lagrange A, Unsicker K (1991) Effects of basic fibroblast growth factor on survival and choline acetyltransferase development of spinal cord neurons. Dev Brain Res 62:257-261.

Grothe C, Meisinger C, Hertenstein A, Kurz H, Wewetzer K (1997) Expression of fibroblast growth factor-2 and fibroblast growth factor receptor-1 messenger RNAs in spinal ganglia and sciatic nerve: regulation after peripheral nerve lesion. Neuroscience 76:123-135.

Guest JD, Hesse D, Schnell L, Schwab ME, Bunge MB, Bunge RP (1997) Influence of IN-1 antibody and acidic FGF-fibrin glue on the response of injured corticospinal tract axons to human Schwann cell grafts. J Neurosci Res 50:888-905.

Gundersen RW, Barrett JN (1980) Characterization of the turning response of dorsal root neurites toward nerve growth factor. J Cell Biol 87:546-554.

Hargreaves K, Dubner R, Brown F, Flores C, Joris J (1988) A new and sensitive method for measuring thermal nociception in cutaneous hyperalgesia. Pain 32:77-88.

Hoyle GW, Mercer EH, Palmiter RD, Brinster RL (1993) Expression of NGF in sympathetic neurons leads to excessive axon outgrowth from ganglia but decreased terminal innervation within tissues. Neuron 10:1019-1034.

Krenz NR, Meakin SO, Krassioukov AV, Weaver LC (1999) Neutralizing intraspinal nerve growth factor blocks autonomic dysreflexia caused by spinal injury. J Neurosci 19:7405-7414.

Kunkel-Bagden E, Dai H-N, Bregman BS (1993) Methods to assess the development and recovery of locomotor function after spinal cord injury in rats. Exp Neurol 119:153-164.

Li Y, Field PM, Raisman G (1997) Repair of adult rat corticospinal tract by transplants of olfactory ensheathing cells. Science 277:2000-2002.

Liuzzi FJ, Lasek RJ (1987) Astrocytes block axonal regeneration in mammals by activating the physiological stop pathway. Science 237:642-645.

McFarlane S, Cornel E, Amaya E, Holt CE (1996) Inhibition of FGF receptor activity in retinal cell axons causes errors in target recognition. Neuron 17:245-254.

Meisinger C, Grothe C (1997) Differential regulation of fibroblast growth factor-2 (FGF-2) and FGF receptor-1 mRNAs and FGF-2 isoforms in spinal ganglia and sciatic nerve after peripheral nerve lesion. J Neurochem 68:1150-1158.

Michael GJ, Kaya E, Averill S, Rattray M, Clary DO, Priestley JV (1997) TrkA-immunoreactive neurons in the rat spinal cord. J Comp Neurol 385:441-455.

Ming G, Song H, Berninger B, Inagaki N, Tessier-Lavigne M, Poo M (1999) Phospholipase $\mathrm{C} \gamma$ and phosphoinositide 3-kinase mediate cytoplasmic signaling in nerve growth cone guidance. Neuron 23:139-148.

Mohajeri MH, Bartsch U, van der Putten H, Sansig G, Mucke L, Schachner M (1996) Neurite outgrowth on nonpermissive substrates in vitro is enhanced by ectopic expression of the neural adhesion molecule L1 by mouse astrocytes. Eur J Neurosci 8:1085-1097.

Nakahara Y, Gage FH, Tuszynski MH (1996) Grafts of fibroblasts genetically modified to secrete NGF, BDNF, NT-3, or basic FGF elicit differential responses in the adult spinal cord. Cell Transplant 5:191-204.

Neumann S, Woolf CJ (1999) Regeneration of dorsal column fibers into and beyond the lesion site following adult spinal cord injury. Neuron 23:83-91.

New HV, Mudge AW (1986) Calcitonin gene-related peptide regulates acetylcholine receptor synthesis. Nature 323:809-811.

Oudega M, Hagg T (1996) Nerve growth factor promotes regeneration of sensory axons into adult rat spinal cord. Exp Neurol 140:218-229.

Pindzola RR, Doller C, Silver J (1993) Putative inhibitory extracellular matrix molecules at the dorsal root entry zone of the spinal cord during development and after root and sciatic nerve lesions. Dev Biol 156:34-48.

Ramer MS, Priestley JV, McMahon SB (2000) Functional regeneration of sensory axons into the adult spinal cord. Nature 403:312-316.

Ramer MS, Duraisingam I, Priestley JV, McMahon SB (2001) Twotiered inhibition of axon regeneration at the dorsal root entry zone J Neurosci 21:2651-2660.

Reier PJ, Stensaas LJ, Guth L (1983) The astrocytic scar as an impediment to regeneration in the central nervous system. In: Spinal cord reconstruction, pp 163-195. New York: Raven.

Romero MI, Smith GM (1998) Adenoviral gene transfer into the normal and injured spinal cord: enhanced transgene stability by combined administration of temperature-sensitive virus and transient immune blockade. Gene Ther 5:1612-1621.

Romero MI, Rangappa N, Li L, Lightfoot S, Garry MG, Smith GM (2000) Extensive sprouting of sensory afferents and hyperalgesia induced by conditional expression of nerve growth factor into the adult spinal cord. J Neurosci 20:4435-4445

Rosenberg GA, Cunningham LA, Wallace J, Alexander S, Estrada EY, Grossetete M, Razhagi A, Miller K, Gearing A (2001) Immunohistochemistry of matrix metalloproteinases in reperfusion injury to the rat brain: activation of MMP-9 linked to stromelysin-1 and microglia in cell culture. Brain Res 893:104-112.

Saffell JL, Williams EJ, Mason IJ, Walsh FS, Doherty P (1997) Expression of a dominant negative FGF receptor inhibits axonal growth and FGF receptor phosphorylation stimulated by CAMs. Neuron 18:231-242.

Schnell L, Schwab ME (1990) Axonal regeneration in the rat spinal cord produced by an antibody against myelin-associated neurite growth inhibitors. Nature 343:269-272.

Schwab ME, Bartholdi D (1996) Degeneration and regeneration of axons in the lesioned spinal cord. Physiol Rev 76:319-370.

Sims TJ, Gilmore SA (1994) Regrowth of dorsal root axons into a radiation-induced glial-deficient environment in the spinal cord. Brain Res 634:113-126.

Smith GM, Romero MI (1999) Adenoviral-mediated gene transfer to enhance neuronal survival, growth, and regeneration. J Neurosci Res $55: 147-157$.

Song H, Ming G-I, Poo M (1997) cAMP-induced switching in turning direction of nerve growth cones. Nature 388:275-279.

Song H, Ming G, He Z, Lehmann M, McKerracher L, Tessier-Lavigne M, Poo M (1998) Conversion of neuronal growth cone responses from repulsion to attraction by cyclic nucleotides. Science $281: 1515-1518$

Varon S, Conner JM (1994) Nerve growth factor in CNS repair. J Neurotrauma 11:473-486.

Williams EJ, Furness J, Walsh FS, Doherty P (1994) Activation of the FGF receptor underlies neurite outgrowth stimulated by L1, N-Cam, and N-cadherin. Neuron 13:583-594.

Yoshida K, Gage FH (1992) Cooperative regulation of nerve growth factor synthesis and secretion in fibroblasts and astrocytes by fibroblast growth factor and other cytokines. Brain Res 569:14-25.

Zolman JF (1993) Biostatistics: experimental design and statistical inference. New York: Oxford UP.

Zuo J, Neubauer D, Dyess K, Ferguson TA, Muir D (1998) Degradation of chondroitin sulfate proteoglycan enhances the neurite-promoting potential of spinal cord tissue. Exp Neurol 154:654-662. 